\title{
Electron acceleration by amplified wakefield generated by two copropagating laser pulses in plasma
}

\author{
Gaurav Raj, Ajay K. Upadhyaya, Rohit K. Mishra, and Pallavi Jha \\ Department of Physics, University of Lucknow, Lucknow 226007, India
}

(Received 21 September 2007; published 28 July 2008)

\begin{abstract}
A detailed analytical theory is developed for studying the phenomenon of generation of high amplitude wakefield using a seed laser pulse and another coaxially propagating, trailing pulse. The two laser pulses are separated by a fixed distance between them and have the same polarization, frequency, length, intensity, and profile. The study shows that the maximum energy gained by the test electron significantly depends upon the distance between the two pulses. Phase space analysis shows that a test electron of significantly lower energy, injected behind the trailing laser pulse, will be trapped and accelerated to higher energy as compared to the single pulse case.
\end{abstract}

DOI: 10.1103/PhysRevSTAB.11.071301

PACS numbers: $52.38 . \mathrm{Kd}$

\section{INTRODUCTION}

Plasma-based accelerators are capable of producing compact and high energy electron sources in much shorter distances than conventional accelerators. This is due to large longitudinal electric field gradients that can be excited without the limitation of breakdown as in radio frequency accelerators. The excitation of plasma waves with large amplitude and a highly relativistic phase velocity can be accomplished by a number of schemes: a beat wave accelerator [1,2] (BWA), a plasma wakefield accelerator [3] (PWFA), a laser wakefield accelerator [1,4,5] (LWFA), and a self-modulated laser wakefield accelerator [6,7] (SM-LWFA). In BWA scheme plasma waves are resonantly excited by beating of two laser pulses with a frequency difference equal to the plasma frequency. The PWFA utilizes a high current bunched electron beam. In LWFA a short, high power laser pulse produces accelerating wakefields as it propagates through plasma. Further, in SM-LWFA the plasma wave is generated through resonant self-modulation [6] of a relatively long pulse of a few plasma oscillation periods, which is usually coupled with Raman forward scattering (RFS).

Recently some innovative concepts have been proposed for generation of high amplitude plasma waves. One such scheme uses two slightly detuned counterpropagating laser pulses, an ultrashort laser pulse, and a long pulse [8]. This can be understood as a kind of Raman seeding. In another scheme, a high amplitude wakefield is generated by a copropagating second laser pulse behind the first pulse [9]. This scheme has been studied using 1D and 2D particle-in-cell (PIC) simulations by considering the first pulse to be an ultrashort pulse (shorter than a plasma oscillation period), while the second pulse is relatively long (few plasma oscillation periods). The second pulse is cross phase modulated (XPM) [9-12] leading to generation of high amplitude wakefields. The advantages of such schemes are that they allow high amplitude plasma wave generation within small plasma dimensions, with moderate laser intensities in a controlled way. Also it releases the necessity of using ultrashort intense laser pulses in the standard LWFA scheme. Further, employing lasers in either a three-pulse or two-pulse configuration [13,14], colliding pulse injector (CPI) concept has been proposed for enhanced trapping and acceleration of background plasma electrons. Recent simulation [14] and experiment [15] based on CPI have reported generation of monoenergetic multi-MeV electron beams.

The aim of the present work is to take up a detailed analytical study of a novel idea of wakefield amplification making use of two laser pulses copropagating one behind the other, with a fixed distance between them. The laser pulses are considered to be identical, such that they have the same moderate intensities, polarizations, frequencies, and profiles. The study is aimed at observing the effect of the electric wakefield generated by the seed (first) laser pulse on the wakefield generated by the trailing (second) laser pulse. The study proceeds by considering plasma to be cold, uniform, and underdense $\left(n_{0} \ll n_{\mathrm{cr}} / 4\right.$, where $n_{0}$ is the unperturbed plasma electron density and $n_{\text {cr }}$ is the critical density required for laser pulse propagation in plasma). The model is applicable for weakly relativistic laser pulses with $a_{0}^{2} \ll 1$, where $a_{0}$ is the laser strength parameter. The pulse length $(L)$ is considered to be short ( $L \leq \lambda_{p}$, where $\lambda_{p}$ is the plasma wavelength), so that large amplitude laser wakefields can be generated. For pulse lengths near the plasma wavelength, relativistic selffocusing of the laser beam does not occur in plasma. Hence, the pulses will propagate over a Rayleigh length (which is of the order of dephasing length) without undergoing any significant distortion due to relativistic nonlinearity $[16,17]$. Further, a one-dimensional (1D) model is used for which broad laser beams $\left(r_{0} \gg \lambda_{p}\right.$, where $r_{0}$ is the minimum spot size of the laser beam) are considered [16]. This also results in a large Rayleigh length $Z_{R}\left(=\pi r_{0}^{2} / \lambda_{0}\right.$, where $\lambda_{0}$ is the laser wavelength) leading to longer acceleration distance in uniform plasma. The present study 
shows that efficient electron acceleration can be achieved in the weakly relativistic, 1D linear wake regime using the two-pulse scheme.

The organization of the paper is as follows: In Sec. II fundamental equations governing wakefield generation by the seed pulse are given and the concept of trailing pulse is introduced. In Sec. III, the effect of pulse separation on the accelerating force experienced by a test electron in the presence of the resultant amplified wakefield is analyzed. The theory of injection and trapping of a test electron in the amplified wakefield generated behind the trailing pulse is studied using phase space analysis. The present case of two coaxial laser pulses is compared with the single pulse case (standard LWFA scheme). Conclusions are presented in Sec. IV.

\section{WAKEFIELD GENERATION AND AMPLIFICATION}

\section{A. Seed and trailing pulse dynamics}

Consider a linearly polarized seed laser pulse propagating through uniform preionized plasma along the positive $z$ direction. The electric field vector of the pulse is given by

$$
\boldsymbol{E}(r, z, t)=\hat{e}_{x} E_{0}(r, z, t) \cos \left(k_{0} z-\omega_{0} t\right),
$$

where $\hat{e}_{x}$ is the unit vector of polarization and $E_{0}(r, z, t)$, $k_{0}$, and $\omega_{0}$ are the amplitude, propagation constant, and frequency of the laser pulse, respectively.

Wakefield generation due to interaction of a laser pulse with plasma (in the weakly relativistic regime) is governed by fluid (Lorentz force and continuity) equations

$$
\begin{aligned}
\frac{\partial \boldsymbol{v}}{\partial t}= & -\frac{e\left(\boldsymbol{E}+\boldsymbol{E}_{w}\right)}{m}-\frac{1}{2} \nabla(\boldsymbol{v} \cdot \boldsymbol{v})+\boldsymbol{v} \\
& \times\left(\nabla \times \boldsymbol{v}-\frac{e\left(\boldsymbol{B}+\boldsymbol{B}_{w}\right)}{m c}\right)
\end{aligned}
$$

and

$$
\frac{\partial n}{\partial t}+\nabla \cdot(n \boldsymbol{v})=0
$$

where $\boldsymbol{v}$ is the velocity of plasma electrons under the influence of the perturbing laser field, $m$ is the rest mass, and $n$ is the density of plasma electrons. Further, $\boldsymbol{E}_{w}$ and $\boldsymbol{B}_{w}$ are the generated electric and magnetic wakefields, respectively, while $\boldsymbol{B}$ is the magnetic field vector of the laser pulse. In order to study the excitation of plasma waves we use the quasistatic approximation (QSA), according to which it is assumed that the duration of interaction between the plasma and the pulse is smaller than the characteristic time of modification of the laser pulse due to nonlinear self-effects [12]. This corresponds to writing the plasma fluid equations in terms of independent variables $\xi\left(=z-v_{g} t\right.$, where $v_{g}$ is the group velocity of the laser pulse) and $\tau(=t)$, and neglecting explicit dependence of the fluid variables on $\tau$. Thus, plasma electrons experience a laser field, which is a function of $\xi$ and $r$ variables only. Perturbative expansion of Eq. (2a) and substitution of Eq. (1) leads to the first order velocity component representing transverse quiver motion of plasma electron as

$$
v_{x}=c a(r, \xi, \tau) \sin k_{0} \xi,
$$

where $a(r, \xi, \tau)=e E_{0}(r, \xi, \tau) / m c \omega_{0}$ is the normalized electric field amplitude of the seed pulse. With the help of Eq. (3), the second order slow axial velocity component can be obtained from

$$
v_{g} \frac{\partial v_{z}}{\partial \xi}=\frac{e}{m} E_{w z}+\frac{1}{4} \frac{\partial}{\partial \xi}\left(a^{2} c^{2}\right),
$$

where $v_{z}\left(E_{w z}\right)$ is the longitudinal component of the second order velocity (generated electric field). Further, it has been assumed that if the electron fluid is initially vortex free then it remains so during subsequent stages of interaction $[\boldsymbol{v} \times[\nabla \times \boldsymbol{v}-(e \boldsymbol{B} / m c)]=0]$.

Propagation of the laser pulse through plasma leads to charge separation between electrons and heavy ions due to ponderomotive force [represented by the second term on right-hand side of Eq. (2a)] of the pulse. As a consequence, electrostatic Coulomb force of attraction appears between electrons and ions, which sets the plasma oscillating at frequency $\omega_{p}\left(=4 \pi e^{2} n_{0} / m\right)$. This leads to plasma wave generation in the wake of the laser pulse. Using QSA, the time dependent Maxwell's equation representing the longitudinal electric wakefield is given by [18]

$$
\frac{v_{g}}{c} \frac{\partial E_{w z}}{\partial \xi}=\frac{4 \pi}{c} J_{z}-\frac{1}{r} \frac{\partial}{\partial r}\left(r B_{w \theta}\right),
$$

where $J_{z}\left(=-e n v_{z}\right)$ is the axial current density. Considering that the $B_{w \theta}$ (magnetic wakefield) component is slowly varying in the transverse direction, the second term on the right-hand side of Eq. (5) can be neglected in comparison to the first. Taking the partial derivative of Eq. (5) with respect to $\xi$ and using Eq. (4) gives

$$
\left[\frac{\partial^{2}}{\partial \xi^{2}}+k_{p}^{2}\right] E_{w z}=-\frac{m c^{2} k_{p}^{2}}{4 e} \frac{\partial a^{2}}{\partial \xi}
$$

where $k_{p}\left(=\omega_{p} / v_{p}\right)$ is the wave number and $v_{p}$ is the phase velocity of the plasma wave. In obtaining Eq. (6), the phase velocity of the plasma wave is considered to be approximately equal to the group velocity of the laser pulse. Generation of axial wakefield in underdense, homogeneous plasma is governed by Eq. (6). Assuming a sinusoidal pulse, its profile can be represented by

$$
a^{2}=a_{r}^{2} \sin ^{2} \pi \xi / L,
$$

where $a_{r}^{2}=a_{0}^{2} \exp \left(-2 r^{2} / r_{0}^{2}\right)$. Using Eq. (7), the solution of Eq. (6) in the region lying behind the pulse $(\xi<0)$ is given by

$$
E_{w z}=\frac{\varepsilon k_{p} f}{8}\left[\sin k_{p}(L-\xi)+\sin k_{p} \xi\right],
$$


where $\varepsilon=m c^{2} a_{r}^{2} / e$ and $f=\left[1-\left(k_{p}^{2} L^{2} / 4 \pi^{2}\right)\right]^{-1}$. Equation (8) represents the axial seed wakefield, as obtained in standard single pulse LWFA schemes $[18,19]$. The field is maximum when $L \rightarrow \lambda_{p}$ and is given by

$$
E_{\substack{w z \\ \max }}=-\frac{\varepsilon \pi^{2}}{4 \lambda_{p}} \cos k_{p} \xi
$$

\section{B. Wakefield amplification}

Consider a second trailing laser pulse copropagating at a distance $\Delta z$ behind the first pulse [Eq. (1)]. The electric field vector of the trailing pulse is given by

$$
\boldsymbol{E}_{t}\left(r, \xi^{\prime}, \tau^{\prime}\right)=\hat{e}_{x} E_{t}\left(r, \xi^{\prime}, \tau^{\prime}\right) \cos k_{0} \xi^{\prime},
$$

where subscript $t$ represents the trailing pulse, $\xi^{\prime}=z-$ $\Delta z-v_{g} t, \tau^{\prime}=t$, and $\xi-\xi^{\prime}=\Delta z$. The trailing pulse is assumed to be propagating coaxially behind the seed pulse. Further, it has the same intensity, polarization, frequency, length, and profile as the seed laser pulse. As the trailing pulse propagates in plasma, electrons experience longitudinal and transverse electrostatic fields generated behind the seed pulse along with the fields due to the second pulse. Consequently, the Lorentz force equation governing the slow plasma electron motion behind the trailing pulse is given by

$$
\frac{\partial \boldsymbol{v}_{t}}{\partial t}=-\frac{e}{m}\left[\boldsymbol{E}_{w}+\boldsymbol{E}_{t w}\right]-\frac{1}{2} \nabla\left(v_{t x}^{2}\right),
$$

where $v_{t x}\left[=c e E_{t}\left(r, \xi^{\prime}, \tau^{\prime}\right) / m c \omega_{0}\right]$ is the quiver velocity imparted to plasma electrons under the influence of the electric field [Eq. (10)] of the trailing pulse. The evolution of the resultant (slow) plasma electron velocity $\left(\boldsymbol{v}_{t}\right)$ is governed by the wakefield $\left(\boldsymbol{E}_{w}\right)$ of the seed laser pulse, the resultant wakefield $\left(\boldsymbol{E}_{t w}\right)$ behind the trailing pulse and the ponderomotive force given by the last term on the righthand side of Eq. (11). Using QSA, the slow axial velocity component as obtained from Eq. (11) is given by

$$
v_{g} \frac{\partial v_{t z}}{\partial \xi^{\prime}}=\frac{e}{m}\left(E_{w z}+E_{t w z}\right)+\frac{1}{4} \frac{\partial}{\partial \xi^{\prime}}\left(c^{2} a_{t}^{2}\right),
$$

where $v_{t z}$ and $E_{t w z}$ are the axial components of velocity and electric wakefields due to trailing laser pulse, respectively. Further, $a_{t}\left(r, \xi^{\prime}, \tau^{\prime}\right)\left[=e E_{t}\left(r, \xi^{\prime}, \tau^{\prime}\right) / m c \omega_{0}\right]$ is the normalized electric field amplitude of the trailing pulse. With the help of Eq. (5) (for trailing pulse) along with Eq. (12) and using the same procedure as used for the seed pulse, the axial component of the electric wakefield due to the trailing pulse is given by

$$
\left[\frac{\partial^{2}}{\partial \xi^{\prime 2}}+k_{p}^{2}\right] E_{t w z}=k_{p}^{2} E_{w z}-\frac{m c^{2} k_{p}^{2}}{4 e} \frac{\partial a_{t}^{2}}{\partial \xi^{\prime}} .
$$

Since in homogeneous plasma the wakefield generated behind the laser pulse is utilized for accelerating properly phased electrons, the solution of Eq. (13) behind $\left(\xi^{\prime}<0\right)$ the trailing pulse is

$$
\begin{aligned}
E_{t w z}= & \frac{f k_{p} \varepsilon}{8}\left[\sin k_{p} L \cos \frac{k_{p} L}{2} \cos k_{p}\left(\left(\xi^{\prime}-\Delta z\right)-\frac{L}{2}\right)\right. \\
& +L k_{p} \sin \frac{k_{p} L}{2} \sin k_{p}\left(\frac{L}{2}-\left(\xi^{\prime}+\Delta z\right)\right) \\
& \left.+2 \sin \frac{k_{p} L}{2} \cos k_{p}\left(\xi^{\prime}-\frac{L}{2}\right)\right] .
\end{aligned}
$$

In obtaining Eq. (14), it is assumed that the trailing pulse has the same profile $\left(a_{t}^{2}=a_{r}^{2} \sin ^{2} \pi \xi^{\prime} / L\right)$ and pulse length $(L)$ as the seed pulse. The resultant axial wakefield behind the trailing pulse will be maximum when the laser pulse length is nearly equal to the plasma wavelength $\left(L \approx \lambda_{p}\right)$. Using Eq. (14) the maximum axial wakefield behind the trailing pulse in the limit $L \rightarrow \lambda_{p}$ is given by

$$
\begin{aligned}
E_{\max }= & \frac{\varepsilon \pi^{2}}{4 \lambda_{p}}\left\{\pi \sin \left[k_{p}\left(\xi^{\prime}+\Delta z\right)\right]-\cos \left[k_{p}\left(\xi^{\prime}-\Delta z\right)\right]\right. \\
& \left.-\cos k_{p} \xi^{\prime}\right\} .
\end{aligned}
$$

It may be noted that the resultant electric wakefield is a function of the interpulse separation $\Delta z$. An appropriate value of this parameter will be required for generating optimum amplitude axial electric wakefields behind the trailing pulse.

\section{ACCELERATION OF A TEST ELECTRON}

Consider a test electron moving along the positive $z$ direction, behind the trailing laser pulse. Because of the axial wakefield [Eq. (15)], the electron will experience a maximum force $\left(F_{t w z}\right)$ given by

$$
F_{t w z}=\frac{d p}{d t}=-e E_{\substack{\text { max } \\ \text { a }}}
$$

where $p$ ( $=m \gamma_{e} v_{e z}, v_{e z}$ is the test electron velocity) is the relativistic momentum and $\gamma_{e}\left\{=\left[1+\left(p^{2} / m^{2} c^{2}\right)\right]^{1 / 2}\right\}$ is the relativistic factor for the test electron. In Fig. 1, using the set of Eqs. (15) and (16), we give the surface plot for evolution of the maximum normalized accelerating force $\left(F_{t w z} / m c \omega_{p}\right)$ acting on a test electron with normalized distance $\left(\xi^{\prime} / \lambda_{p}\right)$ for different values of normalized interpulse separation $\left(\Delta z / \lambda_{p}\right)$. The parameters used in plotting Fig. 1 are $a_{0}^{2}=0.1, \quad \lambda_{0}=1.0 \mu \mathrm{m}, \quad \omega_{0}=1.88 \times$ $10^{15} \mathrm{sec}^{-1}$, laser pulse intensity $I_{0}=1.4 \times 10^{17} \mathrm{~W} / \mathrm{cm}^{2}$, $r_{0}=25.0 \mu \mathrm{m}, \quad r=4.0 \mu \mathrm{m}, \quad n_{0}=5.32 \times 10^{18} \mathrm{~cm}^{-3}$, $\lambda_{p}=15.0 \mu \mathrm{m}$, and $\omega_{p}=1.25 \times 10^{14} \mathrm{sec}^{-1}$. The figure shows that, for a given value of $\Delta z / \lambda_{p}$, the accelerating force evolves periodically with the distance. It is observed that, as the distance $\Delta z$ between the seed and the trailing pulse increases, the normalized force oscillates with increasing amplitude. The two peaks seen in the figure occur at $\Delta z / \lambda_{p}=0.40$ and 0.85 for which accelerating gradients are calculated to be 30 and $42 \mathrm{GV} / \mathrm{m}$, respectively. Since the maximum energy gained by the test electron depends 


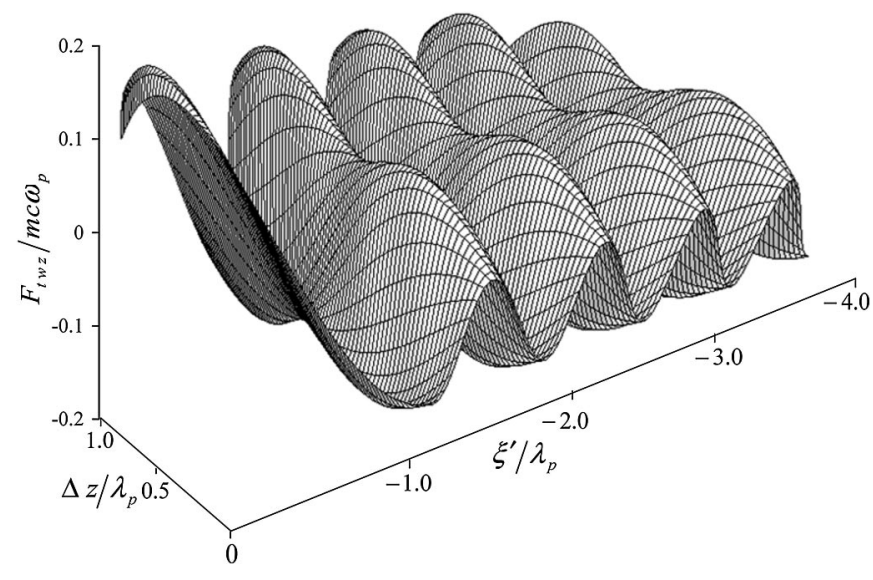

FIG. 1. Variation of peak normalized accelerating force $\left(F_{t w z} / m c \omega_{p}\right)$ acting on a test electron with normalized distance $\left(\xi^{\prime} / \lambda_{p}\right)$ for different values of normalized interpulse separation $\left(\Delta z / \lambda_{p}\right)$ in the two-pulse case for $a_{0}^{2}=0.1, \lambda_{p}=15.0 \mu \mathrm{m}$, $r_{0}=25.0 \mu \mathrm{m}$, and $r=4.0 \mu \mathrm{m}$.

directly on the peak accelerating force experienced by it due to the wakefield, the figure shows that maximum enhancement of electron energy will occur for $\Delta z$ close to $\lambda_{p}$.

The exchange of energy between the plasma wave and test electron can be obtained with the help of Eq. (16) as

$$
\frac{d \gamma_{e}}{d t}=-\frac{e v_{e z}}{m c^{2}} E_{\substack{t w z \\ \text { max }}}=-\frac{e}{m c}\left[1-\frac{1}{\gamma_{e}^{2}}\right]^{1 / 2} E_{t w z} .
$$

In obtaining Eq. (17) transverse velocity components of the test electron have been neglected in comparison to the axial velocity.

Defining the phase of the test electron relative to the resultant wakefield generated behind the trailing pulse as $\psi$ $\left(=k_{p} \xi^{\prime}+k_{p} \Delta z\right)$, Eq. (15) can be rewritten as

$$
E_{\substack{\max \\ \max }}=\frac{\varepsilon \pi^{2}}{4 \lambda_{p}}\left[\pi \sin \psi-\cos \left(\psi-2 k_{p} \Delta z\right)-\cos \left(\psi-k_{p} \Delta z\right)\right] .
$$

Differentiating $\psi$ with respect to time gives

$$
\frac{d \psi}{d t}=k_{p} v_{e z}-\omega_{p}=k_{p} c\left[1-\frac{1}{\gamma_{e}^{2}}\right]^{1 / 2}-\omega_{p} .
$$

Solving Eqs. (17) and (18b), using Eq. (18a) and integrating gives

$$
\begin{aligned}
\gamma_{e}-\beta_{p}\left(\gamma_{e}^{2}-1\right)^{1 / 2}= & A\left[\pi \cos \psi+\sin \left(\psi-k_{p} \Delta z\right)\right. \\
& \left.+\sin \left(\psi-2 k_{p} \Delta z\right)\right]+C,
\end{aligned}
$$

where $A=\left(\pi a_{0}^{2} / 8\right) \exp \left(-2 r^{2} / r_{0}^{2}\right), \quad \beta_{p}=\omega_{p} / c k_{p}, C=$ $\gamma_{e 0}-\beta_{p}\left(\gamma_{e 0}^{2}-1\right)^{1 / 2}-A\left\lfloor\pi-\sin \left(k_{p} \Delta z\right)-\sin \left(2 k_{p} \Delta z\right)\right\rfloor$ is the constant of integration, and $\left(\gamma_{e 0}-1\right) m c^{2}$ is the test electron energy at the initial phase.
For the single pulse case the maximum axial field [Eq. (9)] can be written as

$$
E_{\text {max }}=-\frac{\varepsilon \pi^{2}}{4 \lambda_{p}} \cos \psi_{s}
$$

where $\psi_{s}\left(=k_{p} \xi\right)$ is the phase of the test electron relative to the wakefield generated by the single pulse. Using Eq. (20) along with Eqs. (17) and (18b) gives

$$
\gamma_{e}-\beta_{p}\left(\gamma_{e}^{2}-1\right)^{1 / 2}=A\left[\sin \psi_{s}\right]+C_{s},
$$

where $C_{s}=\gamma_{e 0 s}-\beta_{p}\left(\gamma_{e 0 s}^{2}-1\right)^{1 / 2}$ is the constant of integration and $\left(\gamma_{e 0 s}-1\right) m c^{2}$ is the test electron energy at the initial phase for the single pulse case.

The trapped and untrapped trajectories of the test electron in the phase space bucket of the resultant wakefield generated by two pulses and a single pulse are, respectively, plotted in Figs. 2 and 3, using Eqs. (19) and (21). The figures are plotted for the same parameters used in plotting Fig. 1 along with $\beta_{p}=0.998$. The set of curves (a) $-(e)$ in Fig. 2 represents the trajectories for electrons injected with initial energies given by $0.30,0.40,0.50,2.60$, and $3.73 \mathrm{MeV}$, respectively, in the phase space bucket at $\psi=0$ for the two-pulse scheme, with $\Delta z=0.40 \lambda_{p}$. Correspondingly, curves $(a)-(e)$ of Fig. 3 represent the orbits for electrons of same initial energies (as in Fig. 2) injected at $\psi_{s}=0$ for the single pulse scheme. The curves $(b)$ and $(d)$ in Figs. 2 and 3, respectively, show the separatrices, which are the orbits that separate the trapped [curves lying inside the curves $(b)$ and $(d)$ ] and untrapped [curves lying outside the curves $(b)$ and $(d)$ ] trajectories of the test electron [20]. It is observed from Fig. 2 that the minimum energy $\left[\left(\gamma_{e 0}-1\right) m c^{2}\right]$ necessary for an electron to trace the separatrix in the phase space is $0.40 \mathrm{MeV}$. However, for

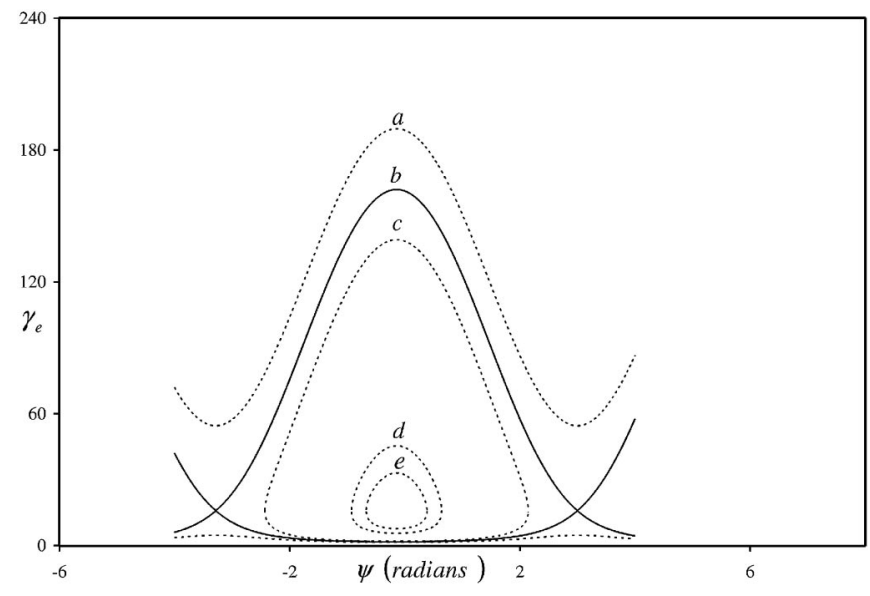

FIG. 2. Phase space plot for the two-pulse case showing untrapped orbit for $0.30 \mathrm{MeV}$ (curve $a$ ), separatrix for $0.40 \mathrm{MeV}$ (curve $b$ ), and trapped orbits for $0.50 \mathrm{MeV}$ (curve $c$ ), $2.60 \mathrm{MeV}$ (curve $d$ ), and $3.73 \mathrm{MeV}$ (curve $e$ ) initial energy electrons injected at $\psi=0$ with $\Delta z=0.40 \lambda_{p}, a_{0}^{2}=0.1, \beta_{p}=0.998$, $\lambda_{p}=15.0 \mu \mathrm{m}, r_{0}=25.0 \mu \mathrm{m}$, and $r=4.0 \mu \mathrm{m}$. 


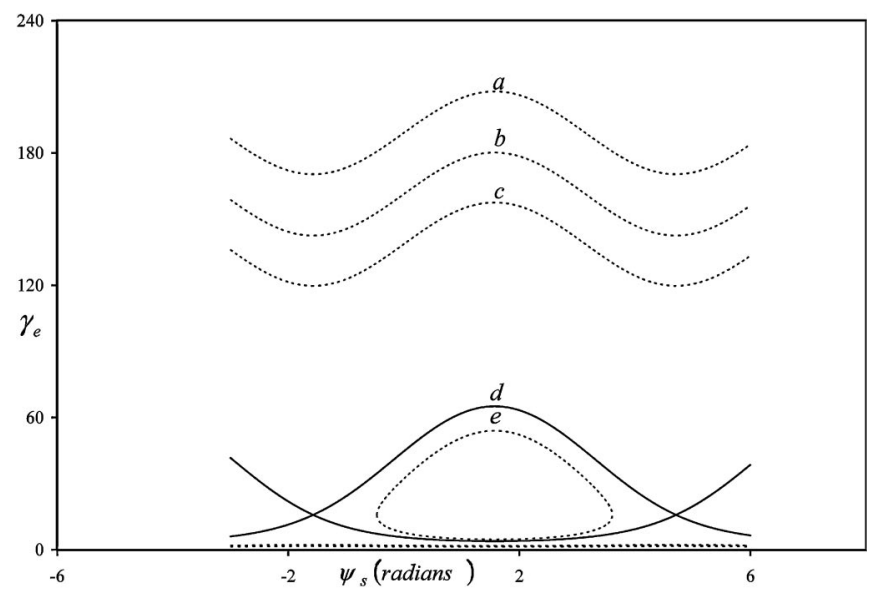

FIG. 3. Phase space plot for the single pulse case showing untrapped orbits for $0.30 \mathrm{MeV}$ (curve $a$ ), $0.40 \mathrm{MeV}$ (curve $b$ ), $0.50 \mathrm{MeV}$ (curve $c$ ), separatrix for $2.60 \mathrm{MeV}$ (curve $d$ ), and trapped orbit for $3.73 \mathrm{MeV}$ (curve $e$ ) initial energy electrons injected at $\psi_{s}=0$ with $a_{0}^{2}=0.1, \beta_{p}=0.998, \lambda_{p}=15.0 \mu \mathrm{m}$, $r_{0}=25.0 \mu \mathrm{m}$, and $r=4.0 \mu \mathrm{m}$.

the single pulse case (Fig. 3) the minimum energy [ $\left(\gamma_{e 0 s}-\right.$ 1) $\left.m c^{2}\right]$ required is $2.60 \mathrm{MeV}$. Consequently, the analysis shows that the minimum injection energy necessary for trapping of the test electron, for the two-pulse case, is approximately 6.5 times less as compared to the single pulse case.

For the two-pulse case (Fig. 2) the electron riding the separatrix [curve $(b)]$ attains a maximum energy $\left[\left(\gamma_{e \max }-\right.\right.$ 1) $\left.m c^{2}\right]$ of $0.09 \mathrm{GeV}$ leading to an energy gain $\left[\left(\gamma_{e \max }-\right.\right.$ $\left.\gamma_{e 0}\right) m c^{2}$ ] of $0.089 \mathrm{GeV}$, while in case of single pulse [Fig. 3, curve $(d)$ ] the maximum energy attained $\left[\left(\gamma_{e s \max }-1\right) m c^{2}\right]$ is $0.03 \mathrm{GeV}$ leading to an energy gain of $\left[\left(\gamma_{e s \max }-\gamma_{e 0 s}\right) m c^{2}\right] 0.027 \mathrm{GeV}$. Thus, the study shows that the energy gained by the $0.40 \mathrm{MeV}$ (injection energy) electron in the two-pulse scheme is approximately 3.0 times more as compared to that attained by the $2.60 \mathrm{MeV}$ (injection energy) electron in the single pulse scheme. The dephasing length $L_{d} \quad\left\{=\lambda_{p} \omega_{0}^{2} /\left[\omega_{p}^{2}+\left(4 c^{2} / r_{0}^{2}\right)\right]\right.$, Ref. [17]\} and the Rayleigh length are approximately 0.3 and $0.2 \mathrm{cms}$, respectively, for the given set of parameters. In uniform plasma, since the Rayleigh length is also the acceleration distance, therefore $L_{d}>Z_{R}$ ensures efficient electron acceleration. It is also observed that the dephasing length for the two cases of interest is approximately the same.

The curves $(d)$ in the Figs. 2 and 3, respectively, represent the closed orbit of the test electron (with injection energy $=2.60 \mathrm{MeV}$ ) trapped in the two-pulse scheme and which will move along the separatrix in the single pulse scheme. In Fig. 2 all closed orbits lying outside (injection energies $<2.60 \mathrm{MeV}$ ) and inside (injection energies $>2.60 \mathrm{MeV}$ ) the curve $(d)$, represent electrons which will, respectively, remain untrapped and trapped in the single pulse scheme. Figure 2 shows that the maximum energy attained by high injection energy $(\geq 2.60 \mathrm{MeV}$ ) electrons is less in the two-pulse case as compared to the single pulse scheme. However, the low injection energy $(<2.60 \mathrm{MeV})$ electrons (untrapped in the single pulse scheme) are now trapped and accelerated to significantly higher energies.

\section{CONCLUSIONS}

The analytical theory presented in this paper suggests a new scheme for generation of high amplitude wakefields (as compared to single pulse LWFA scheme). In the present scheme two identical laser pulses propagate coaxially in homogenous plasma with a given interpulse separation. The study takes into account the effect of electric wakefield generated by the seed laser pulse on the wakefield generated by the trailing laser pulse. It is observed that the maximum energy gained by the test electron behind the trailing pulse significantly depends upon the interpulse separation and it tends to maximize when $\Delta z$ is close to $\lambda_{p}$. Further, phase space analysis clearly shows that under the present scheme a properly phased test electron having significantly lower injection energy can be trapped and accelerated to larger energy as compared to single pulse schemes. In the present two-pulse scheme the maximum energy gained (for the given parameters) by the test electron is of the order of that observed in recent simulation and experiment $[14,15]$ on CPI concept. The two-pulse scheme has the advantage of significantly controlled nonlinearity during the acceleration process as compared to the single pulse scheme, where high intensities $\left(a_{0}^{2} \geq 1\right)$ are a compulsion. The two-pulse scheme in the limit $a_{0}^{2} \rightarrow 1$ can lead to accelerating gradients of hundreds of $\mathrm{GV} / \mathrm{m}$, thus resulting in accelerated electrons with energies characteristic of the single pulse LWFA scheme in the $a_{0}^{2} \geq 1$ regime. Such large accelerating gradients can also be achieved by use of multiple (more than two) laser pulses in the present scheme.

\section{ACKNOWLEDGMENTS}

This work has been done with the financial support of science and engineering research council, department of science and technology, government of India. The authors thank the organization for funding the research project.

[1] T. Tajima and J.M. Dawson, Phys. Rev. Lett. 43, 267 (1979).

[2] C. Clayton et al., Phys. Rev. Lett. 54, 2343 (1985); Y. Kitagawa et al., ibid. 68, 48 (1992); C. Clayton et al., ibid. 70, 267 (1994).

[3] P. Chen, J.M. Dawson, R. W. Huff, and T. Katsouleas, Phys. Rev. Lett. 54, 693 (1985).

[4] L. M. Gorbunov and V. I. Kirsanov, Zh. Eksp. Teor. Fiz. 93, 509 (1987) [Sov. Phys. JETP 66, 290 (1987)]; P. Sprangle et al., Appl. Phys. Lett. 53, 2146 (1988). 
[5] C. Geddes, C. Toth, J. Van Tilborg, E. Esarey, C. B. Schroeder, D. Bruhwiler, C. Nieter, J. Cary, and W. P. Leemans, Nature (London) 431, 538 (2004).

[6] P. Sprangle et al., Phys. Rev. Lett. 69, 2200 (1992); J. Krall et al., Phys. Rev. E 48, 2157 (1993); E. Esarey et al., Phys. Fluids B 5, 2690 (1993).

[7] N. E. Andreev et al., Pis'ma Zh. Eksp. Teor. Fiz. 55, 551 (1992) [JETP Lett. 55, 571 (1992)]; T. Antonsen, Jr. and P. Mora, Phys. Rev. Lett. 69, 2204 (1992).

[8] G. Shvets, N. J. Fisch, A. Pukhov, and J. Meyer-ter-Vehn, Phys. Rev. E 60, 2218 (1999).

[9] E. Esarey, A. Ting, and P. Sprangle, Phys. Rev. A 42, 3526 (1990).

[10] Z. M. Sheng, K. Mima, Y. Sentoku, K. Nishihara, and J. Zhang, Phys. Plasmas 9, 3147 (2002).

[11] G. Bonnaud, D. Teychenne, and J.L. Bobin, Phys. Rev. E 50, R36 (1994).

[12] S. Dalla and M. Lontano, Phys. Rev. E 49, R1819 (1994).
[13] E. Esarey, R. F. Hubbard, W. P. Leemans, A. Ting, and P. Sprangle, Phys. Rev. Lett. 79, 2682 (1997).

[14] G. Fubiani, E. Esarey, C. B. Schroeder, and W. P. Leemans, Phys. Rev. E 73, 026402 (2006).

[15] J. Faure, C. Rechatin, A. Norlin, F. Burgy, A. Tafzi, J. P. Rousseau, and V. Malka, Plasma Phys. Controlled Fusion 49, B395 (2007).

[16] P. Sprangle, E. Esarey, and A. Ting, Phys. Rev. Lett. 64, 2011 (1990).

[17] P. Sprangle, B. Hafizi, J. R. Penano, R. F. Hubbard, A. Ting, C. I. Moore, D. F. Gordon, A. Zigler, D. Kaganovich, and T. M. Antonsen, Jr., Phys. Rev. E 63, 056405 (2001).

[18] Pallavi Jha, Punit Kumar, Ajay K. Upadhyay, and Gaurav Raj, Phys. Rev. ST Accel. Beams 8, 071301 (2005).

[19] E. Esarey, A. Ting, P. Sprangle, and G. Joyce, Comments Plasma Phys. Control. Fusion 12, 191 (1989).

[20] E. Esarey, P. Sprangle, J. Krall, and A. Ting, IEEE Trans. Plasma Sci. 24, 252 (1996). 